\title{
Knockdown of long non-coding RNA HOTAIR sensitizes hepatocellular carcinoma cell to cisplatin by suppressing the STAT3/ABCB1 signaling pathway
}

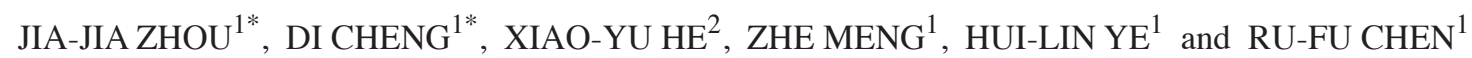 \\ ${ }^{1}$ Guangdong Provincial Key Laboratory of Malignant Tumor Epigenetics and Gene Regulation, \\ Sun Yat-Sen Memorial Hospital, Sun Yat-Sen University, Guangzhou, Guangdong 510120; ${ }^{2}$ Laboratory of Biomechanics \\ and Physiology, Guangdong Provincial Institute of Sports Science, Guangzhou, Guangdong 510663, P.R. China
}

Received August 25, 2016; Accepted August 22, 2017

DOI: $10.3892 / \mathrm{ol} .2017 .7237$

\begin{abstract}
Long non-coding RNA HOX transcript antisense RNA (HOTAIR) has been demonstrated to exhibit oncogenic activity in several types of cancer, including hepatocellular carcinoma (HCC). However, the association between HOTAIR and HCC multidrug resistance remains uncertain. The present study aimed to investigate the role of HOTAIR in HCC chemoresistance; it was found that knockdown of HOTAIR expression in HCC Huh7 cells resulted in decreased cell proliferation and increased chemosensitivity to cisplatin. Furthermore, expression levels of ATP binding cassette subfamily B member 1 (ABCB1) mRNA and protein were decreased in Huh7 cells upon HOTAIR-knockdown. In addition, HOTAIR-knockdown reduced the levels of phosphorylated signal transducer and activator of transcription 3 (STAT3), and inhibition of STAT3 phosphorylation reduced HOTAIR-mediated ABCB1 expression. Together, these findings indicated that knockdown of HOTAIR in Huh7 cells decreased STAT3 activity and ABCB1 expression, and increased chemosensitivity to cisplatin. Thus HOTAIR could serve as a novel potential therapeutic target to reverse multidrug resistance in HCC.
\end{abstract}

\section{Introduction}

Hepatocellular carcinoma (HCC) is the fifth most common malignant tumor and the third leading cause of

Correspondence to: Dr Jia-Jia Zhou, Guangdong Provincial Key Laboratory of Malignant Tumor Epigenetics and Gene Regulation, Sun Yat-sen Memorial Hospital, Sun Yat-sen University, 107 West Yan Jiang Road, Guangzhou, Guangdong 510120, P.R. China

E-mail: jiajiazh2004@126.com

*Contributed equally

Key words: hepatocellular carcinoma, HOX transcript antisense RNA, cisplatin, multidrug resistance, ATP binding cassette subfamily B member 1, signal transducer and activator of transcription 3 signaling cancer-associated mortality worldwide $(1,2)$. There are a variety of $\mathrm{HCC}$ treatment modalities, and chemotherapy is an important and irreplaceable therapeutic strategy for the majority of patients with HCC (3). Cisplatin is a common chemotherapeutic agent used for HCC treatment. However, cancer cells may develop multidrug resistance (MDR) against chemotherapeutic agents, including cisplatin, which leads to the failure of chemotherapy (4). There are a number of mechanisms involved in MDR. One form of MDR is caused by the overexpression of ATP-binding cassette sub-family B member 1 (ABCB1), which pumps anticancer agents out of cells, contributing to a reduced intracellular drug concentration and cytotoxicity $(5,6)$. Inhibition of $\mathrm{ABCB} 1$ expression is able to reduce the resistance of HCC cells to anticancer agents (6). Therefore, identification of the mechanism of action and identification of novel targets for inhibiting ABCB1 may aid the reversal of MDR in HCC.

Long non-coding RNAs (lncRNAs) are non-protein-coding transcripts $>200$ nucleotides. Within the past 10-15 years, lncRNAs have gained the widespread attention of researchers, as they are frequently dysregulated in a variety of human diseases, including cancer, and have critical roles in regulating various biological processes $(7,8)$. HOX transcript antisense RNA (HOTAIR), is located within the homeobox C (HOXC) gene cluster on chromosome 12, which interacts with polycomb repressive complex (PRC2) to enhance the trimethylation of lysine 27 of histone $\mathrm{H} 3$ (H3K27), leading to the repression of multiple genes $(8,9)$. HOTAIR was initially identified to be upregulated, and to promote invasiveness and metastasis in breast cancer (8). In addition, HOTAIR was demonstrated to promote proliferation and reduce apoptosis in pancreatic cancer, colorectal cancer, and HCC (10-12). Overexpression of HOTAIR was positively associated with poor prognosis, tumor progression and recurrence in $\mathrm{HCC}(13,14)$. A recent study reported that HOTAIR also promoted the malignant growth of human liver cancer stem cells (15). A number of studies implicated that HOTAIR has important roles in the tumorigenesis of HCC (12-15). However, the association between HOTAIR and HCC MDR remains unclear.

The present study investigated the potential role of HOTAIR in the regulation of MDR in HCC. HOTAIR small interfering 
RNA (siRNA) was used to silence HOTAIR expression in human Huh7 HCC cells and then the chemosensitivity of Huh7 cells to cisplatin was examined. Knockdown of HOTAIR resulted in decreased cell proliferation and increased chemosensitivity to cisplatin. Furthermore, the increased chemosensitivity of Huh7 cells to cisplatin was associated with decreased expression levels of ABCB1 mRNA and protein when HOTAIR was knocked down. In addition, inhibition of signal transducer and activator of transcription 3 (STAT3) reduced HOTAIR-mediated ABCB1 expression. These results indicate that HOTAIR may have a particular important role in regulation of the chemosensitivity of human HCC cells. The findings of the present study provide a novel insight into the mechanism of HOTAIR in regulating MDR of HCC.

\section{Materials and methods}

Cell line and culture. The Human Huh7 HCC cell line was purchased from the American Type Culture Collection (ATCC, Manassas, VA, USA). Cells were maintained in RPMI-1,640 medium (Gibco; Thermo Fisher Scientific, Inc., Waltham, MA, USA) supplemented with $10 \%$ fetal bovine serum (FBS) (Gibco; Thermo Fisher Scientific, Inc.) at $37^{\circ} \mathrm{C}$ in a humid atmosphere with $5 \% \mathrm{CO}_{2}$. The culture medium was changed every 24 h. AG490 (Sigma-Aldrich; Merck KGaA, Darmstadt, Germany) was used as an inhibitor of STAT3 (16), at the final concentrations of $25 \mu \mathrm{M}$ for $1 \mathrm{~h}$ at room temperature prior to siRNA transfection.

HOTAIR siRNA transfection. Knockdown of human HOTAIR (National Center for Biotechnology Information gene ID, 100,124,700) was achieved using transfection with siRNAs. The siRNA sequences were as follows: HOTAIR, 5'-UAA CAAGACCAGAGAGCUGUU-3'; negative control (NC), 5'-TTCTCCGAACGTGTCACGT-3'. The siRNA were synthesized by GenePharma (GenePharma Co, Ltd, Shanghai, China). Cells were transfected with $100 \mathrm{nM}$ siRNA duplexes using Lipofectamine ${ }^{\mathrm{TM}} 2000$ (Invitrogen; Thermo Fisher Scientific, Inc., Waltham, MA, USA) following manufacturer's protocols. The mock transfected cells were transfected with Lipofectamine 2000 without siRNA (17). Following transfection, cells were incubated in a humidified chamber supplemented with $5 \% \mathrm{CO}_{2}$ at $37^{\circ} \mathrm{C}$. At $48 \mathrm{~h}$ after transfection, cells were harvested for RT-qPCR and Western blotting assay.

Reverse transcription-quantitative polymerase chain reaction (RT-qPCR). RT-qPCR was performed to determine expression of lncRNA HOTAIR (12), and mRNA expression of ABCB1 (16). Briefly, at $48 \mathrm{~h}$ after transfection, cells were harvested in TRIzol Reagent (Invitrogen; Thermo Fisher Scientific, Inc.) following the manufacturer's protocols. Total RNA was reverse transcribed into cDNA using PrimeScript ${ }^{\mathrm{TM}}$ RT Reagent kit (Takara Biotechnology Co., Ltd., Dalian, China). RT-qPCR amplification reactions were performed with SYBR ${ }^{\circledR}$ Premix Ex Taq ${ }^{\mathrm{TM}}$ II (Takara Biotechnology Co., Ltd.). RT-qPCR was performed on CFX96 Real-Time PCR Detection system (Bio-Rad Laboratories, Inc., Hercules, CA, USA). Thermocycling conditions were as follows: Initial denaturation at $95^{\circ} \mathrm{C}$ for $5 \mathrm{~min}$, followed by 40 cycles of denaturation at $95^{\circ} \mathrm{C}$ for $20 \mathrm{sec}$, annealing at $58^{\circ} \mathrm{C}$ for $30 \mathrm{sec}$ and elongation at $72^{\circ} \mathrm{C}$ for $30 \mathrm{sec}$. All fold changes were calculated using the comparative $\mathrm{Cq}\left(2^{-\Delta \Delta \mathrm{Cq}}\right)$ method using GAPDH for normalization (18). Primer sequences used in this study were as follows: ABCB1 forward, 5'-CTTCAGGGTTTCACATTT GGC-3' and reverse, 5'-GGTAGTCAATGCTCCAGTGG-3'; GAPDH forward, 5'-GCACCGTCAAGGCTGAGAAC-3' and reverse, 5'-TGGTGAAGACGCCAGTGGA-3'.

Western blotting assay. At $48 \mathrm{~h}$ after transfection, cells and supernatant of each transfection group were collected. The cells were lysed with radioimmunoprecipitation assay lysis buffer (Beyotime Institute of Biotechnology, Nantong, China) in the presence of protease/phosphatase inhibitor cocktail (Cell Signaling Technology, Inc., Danvers, MA, USA) at $4^{\circ} \mathrm{C}$ for $30 \mathrm{~min}$ and the lysate was centrifuged at $12,000 \mathrm{x} \mathrm{g}$, $4^{\circ} \mathrm{C}$ for $20 \mathrm{~min}$. The concentration of protein was measured using a Bicinchoninic Acid Protein Assay kit (Thermo Fisher Scientific, Inc.). Equal amounts (30 $\mu \mathrm{g}$ per lane) of extracted protein samples were separated by $10 \%$ SDS-PAGE and transferred onto polyvinylidene difluoride membranes (EMD Millipore, Billerica, MA, USA). Following blocking with 5\% non-fat milk for $1 \mathrm{~h}$ at room temperature, the membranes were incubated with the following primary antibodies at $4^{\circ} \mathrm{C}$ under gentle agitation overnight: ABCB1 (1:1,000; ab155421; Abcam, Cambridge, UK), pSTAT3 (1:1,000; \#9145; Cell Signaling Technology, Inc.), STAT3 (1:1,000; \#9,139; Cell Signaling Technology, Inc.) and GAPDH (1:5,000; sc-47,724; Santa Cruz Biotechnology, Inc., Dallas, TX, USA). Following washing with TBST, membranes were incubated with horseradish peroxidase-conjugated secondary antibody (1:5,000; \#1,662,408; Bio-Rad Laboratories, Inc.) for $1 \mathrm{~h}$ at room temperature. The Supersignal ${ }^{\circledR}$ West Pico Chemiluminescent substrate (Thermo Fisher Scientific, Inc.) was used to visualize protein bands on X-ray films, and ImageJ software version 1.5 (National Institutes of Health, Bethesda, MD, USA) gel analysis was used to quantify the protein bands from western blotting films.

Cell migration assay. Cell migration was tested using a Transwell chamber assay. In total, $1.0 \times 10^{5}$ cells were seeded on an $8-\mu \mathrm{m}$ pore size Transwell (Merck KGaA) in serum-free RPMI-1640 medium (Gibco; Thermo Fisher Scientific, Inc.), while RPMI-1,640 medium (Gibco; Thermo Fisher Scientific, Inc.) containing 10\% FBS (Gibco; Thermo Fisher Scientific, Inc.) was placed in the lower well. After incubation at $37^{\circ} \mathrm{C}$ for $48 \mathrm{~h}$, non-migrating cells in the upper chamber surface were removed, migration cells were stained with the Three Step Stain Set kit (Thermo Fisher Scientific, Inc.) for $30 \mathrm{~min}$ at room temperature. The number of migrated cells was counted in 5 randomly selected fields using a light microscope at a magnification of $\mathrm{x} 200$.

Cell viability assay. Cell viability assay was performed by using the Cell Counting kit-8 (CCK-8; Dojindo Molecular Technologies, Inc., Kumamoto, Japan) method. Briefly, cells were seeded in 96-well plates (Corning Incorporated, Corning, NY, USA). Following overnight culture, Huh7 cells were transfected with siHOTAIR or control siRNA for $24 \mathrm{~h}$, then exposed to cisplatin (Selleck Chemicals, Houston, TX, USA) at different final concentrations $(0.1,0.25,0.5,1.0,2.5$, 

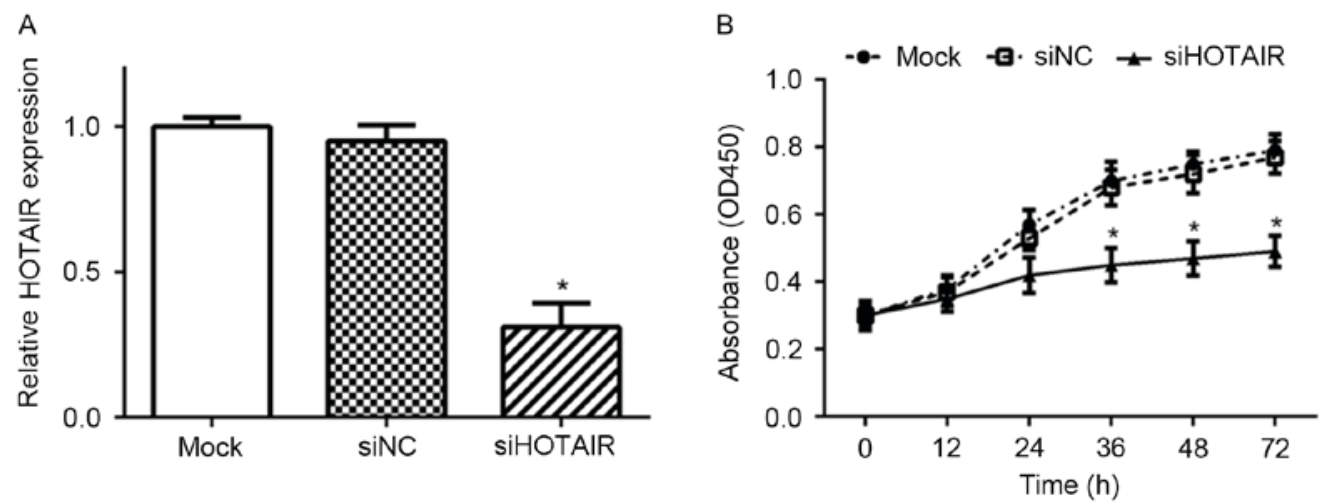

Figure 1. Knockdown of HOTAIR inhibited Huh7 cell proliferation. (A) HOTAIR expression in Huh7 cells transfected with siHOTAIR or siNC, or mock cells, were detected by reverse transcription-quantitative polymerase chain reaction (" $\mathrm{P}<0.05$ vs. siNC); (B) Cell proliferation of Huh7 cells was tested by Cell Counting kit- 8 assay ("P<0.05 vs. siNC). siHOTAIR, small interfering RNA targeted at HOX transcript antisense RNA; NC, negative control.

$5 \mu \mathrm{mol} / \mathrm{l}$ ) for $48 \mathrm{~h}$ in a $\mathrm{CO}_{2}$ incubator, after which the viability was accessed. The CCK-8 assay was used to detect the chemosensitivity of cells according to the manufacturer's protocol. The absorbance at $450 \mathrm{~nm}$ was measured using a microplate reader. A total of six replicate wells were used for each group.

Statistical analysis. Results are presented as mean of three independent experiments \pm standard deviation. Statistical analyses were performed using one-way analysis of variance and LSD t-test to compare between multiple groups and Student's t-test to compare between two groups using GraphPad Prism (version 4.0; GraphPad Software Inc., La Jolla, CA, USA). $\mathrm{P}<0.05$ was considered to indicate a statistically significant difference.

\section{Results}

Knockdown of HOTAIR by specific siRNA. To investigate the role of HOTAIR in HCC cells, specific HOTAIR siRNA (siHOTAIR) was transfected into the Huh7 cell line using Lipofectamine 2000. As presented in Fig. 1, transfection of Huh7 cells with siHOTAIR resulted in significant knockdown of HOTAIR expression. Furtheremore, NC siRNA (siNC) had no significant impact on HOTAIR expression level compared with mock cells.

Knockdown of HOTAIR inhibits the proliferation of Huh7 cells. To examine the role of HOTAIR on prolferation of Huh7 cells, the effect of siHOTAIR on cell growth was assessed. As presented in Fig. 1, the proliferative capacity of Huh7 cells transfected with siHOTAIR was significantly decreased, as compared with siNC and mock cells $(\mathrm{P}<0.05)$.

Knockdown of HOTAIR inhibits the migration of Huh7 cells. The results of Transwell cell migration assay are presented in Fig. 2. Knockdown of HOTAIR significantly inhibited the migration ability of Huh7 cells with $47.83 \pm 6.55$ cells invaded, whereas $107.7 \pm 6.32$ and $104.6 \pm 6.93$ migrating cells were observed in mock, and siNC cells, respectively ( $\mathrm{P}<0.05$, Fig. 2 ).

Knockdown of HOTAIR sensitizes Huh7 cells to cisplatin. To evaluate the effect of HOTAIR on chemosensitivity of Huh7 cells, the viability of Huh7 cells transfected with siHOTAIR and then exposed to cisplatin were assessed by CCK-8 assay. As presented in Fig. 3, Huh7 cells transfected with siHOTAIR were more sensitive to cisplatin than siNC and mock cells, indicating that knockdown of HOTAIR enhanced the sensitivity of Huh7 cells to cisplatin.

Knockdown of HOTAIR reduces the expression of ABCB1 in Huh7 cells. To evaluate the effect of HOTAIR on cisplatin sensitivity of Huh7 cells further, the expression of ABCB1, an important mediator of MDR, was detected. As presented in Fig. 3, ABCB1 expression was positively associated with HOTAIR expression. Compared with siNC and mock cells, the expression levels of $\mathrm{ABCB} 1 \mathrm{mRNA}$ and protein were significantly lower in siHOTAIR cells $(\mathrm{P}<0.05)$.

Knockdown of HOTAIR inhibits the expression of phosphorylated ( $p$ )-STAT3 in Huh7 cells. Since STAT3 has been reported to be involved in the regulation of ABCB1 expression (19), whether HOTAIR regulated ABCB1 expression via the STAT3 signaling was investigated. As demonstrated in Fig. 4, levels of p-STAT3 was significantly decreased in siHOTAIR cells $(\mathrm{P}<0.05)$; in addition, the expression of total STAT3 was not significantly affected in siHOTAIR cells, compared with siNC and mock cells.

Inhibition of STAT3 reduces HOTAIR-mediated ABCBI expression. To further investigate the effect of STAT3 signaling on HOTAIR-mediated ABCB1 expression, Huh7 cells were treated with AG490, a STAT3 inhibitor (16), for $1 \mathrm{~h}$ prior to siRNA transfection. As presented in Fig. 5, levels of ABCB1 and pSTAT3 were significantly reduced in the AG490-treated group (transfected with either with siHOTAIR or siNC), but the suppresion was more evident in the siHOTAIR group. Overall, the data indicated that HOTAIR mediated ABCB1 expression and chemoresistance to cisplatin through the STAT3 signaling pathway in HCC.

\section{Discussion}

MDR of cancer cells is one of primary reasons for the failure of HCC chemotherapy and its suppression may increase the 
A

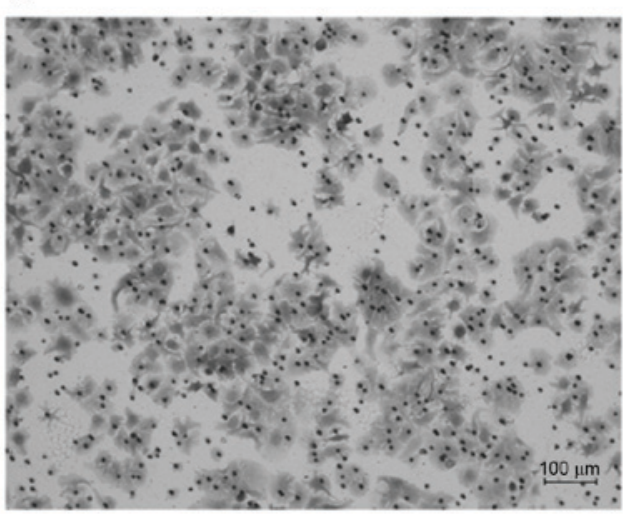

C

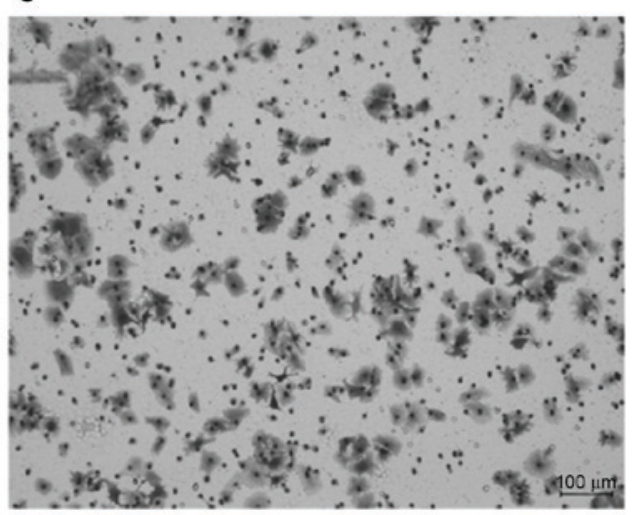

B

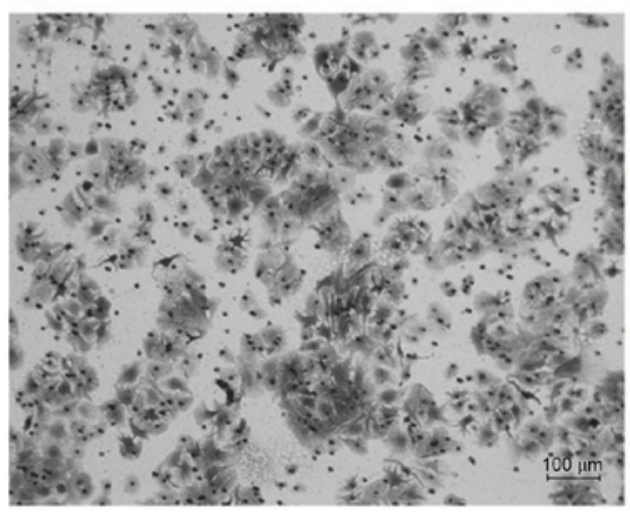

D

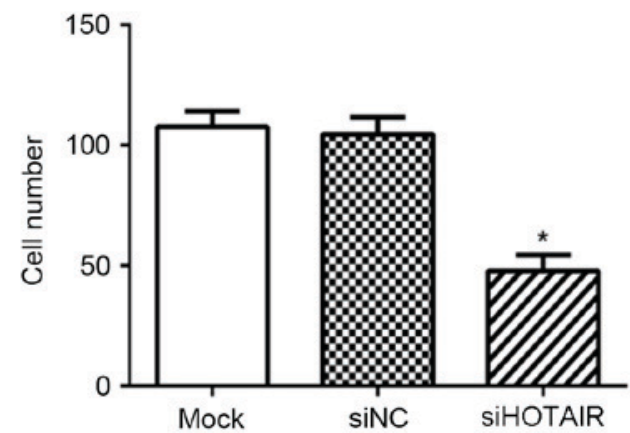

Figure 2. Knockdown of HOTAIR inhibited Huh7 cell migration. Cell migration of Huh7 cells transfected with (A) mock cells, (B) siNC or (C) siHOTAIR were detected by Transwell assay. (D) The number of migrating cells was compared ("P $<0.05$ ). siHOTAIR, small interfering RNA targeted at HOX transcript antisense RNA; NC, negative control.

A

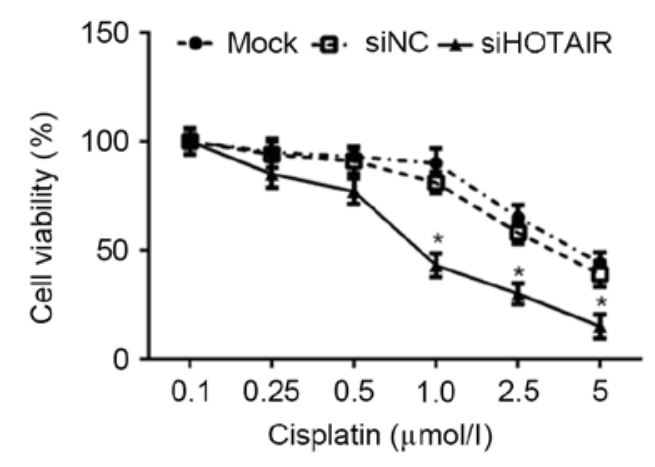

C

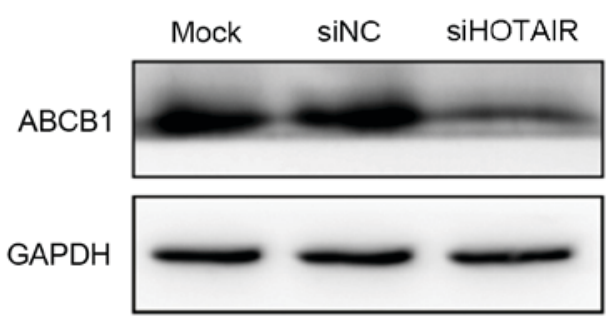

B

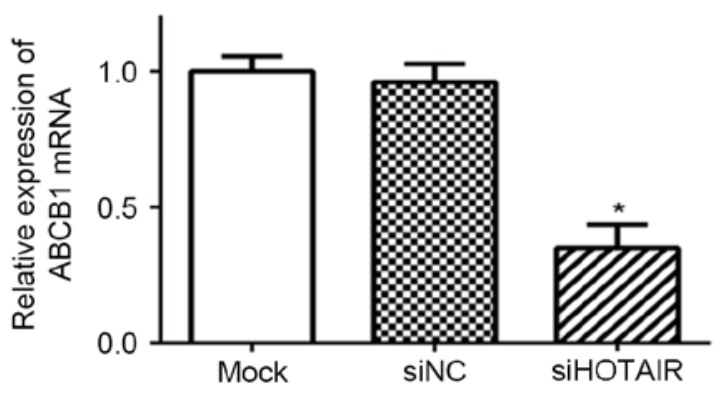

D

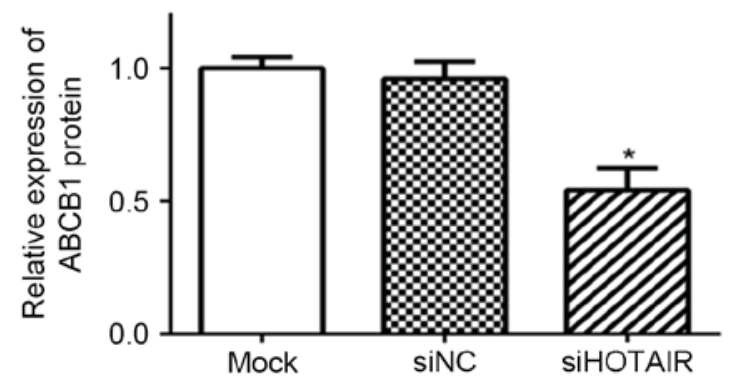

Figure 3. Knockdown of HOTAIR sensitized Huh7 cells to cisplatin and reduced ABCB1 expression. (A) Huh7 cells transfected with siHOTAIR or siNC were treated with cisplatin $(0.1,0.25,0.5,1.0,2.5$ or $5.0 \mu \mathrm{mol} / \mathrm{l})$ for $48 \mathrm{~h}$. Cell viability was assessed by Cell Counting kit- 8 assay ("P<0.05 vs. siNC). Expression levels of ABCB1 (B) mRNA and (C and D) protein in Huh7 cells transfected with siHOTAIR or siNC, or mock cells was detected by reverse transcription-quantitative polymerase chain reaction and western blot analysis, respectively (" $\mathrm{P}<0.05$ vs. siNC). siHOTAIR, small interfering RNA targeted at HOX transcript antisense RNA; NC, negative control; ABCB1, ATP binding cassette subfamily B member 1. 

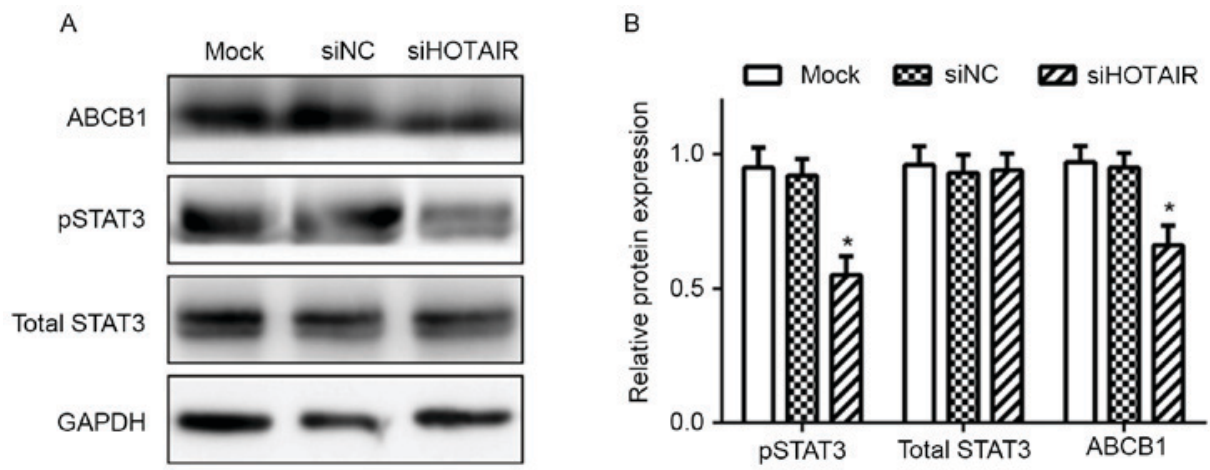

Figure 4. Knockdown of HOTAIR inhibited expression of pSTAT3 in Huh7 cells. (A) Representative image and (B) quantification of expression levels of phosphorylated STAT3, total STAT3 and ABCB1 protein in Huh7 cells transfected with siHOTAIR or siNC, or mock cells were detected by western blot analysis ("P<0.05 vs. siNC). siHOTAIR, small interfering RNA targeted at HOX transcript antisense RNA; NC, negative control; ABCB1, ATP binding cassette subfamily B member 1; pSTAT3, phosphorylated signal transducer and activator of transcription 3 .

A

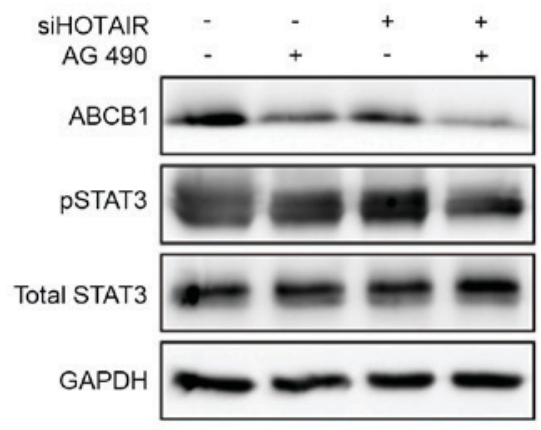

B

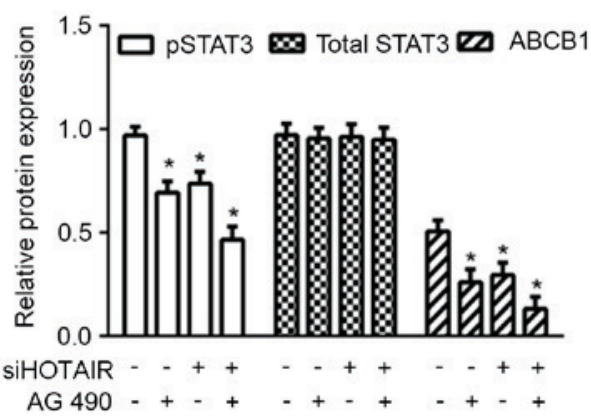

Figure 5. Inhibition of STAT3 reduced HOTAIR-mediated ABCB1 expression in Huh7 cells. (A) Representative image and (B) quantification of Huh7 cells treated with the STAT3 inhibitor AG490 for $1 \mathrm{~h}$ before siHOTAIR or siNC transfection, expression levels of phosphorylated STAT3, total STAT3 and ABCB1 protein in Huh7 cells were detected by western blot analysis ("P<0.05). siHOTAIR, small interfering RNA targeted at HOX transcript antisense RNA; NC, negative control; ABCB1, ATP binding cassette subfamily B member 1; pSTAT3, phosphorylated signal transducer and activator of transcription 3.

efficacy of chemotherapy. A previous study confirmed that inhibition of homeobox transcription factor NANOG reduced ABCB1 expression, leading to the enhanced chemosensitivity to doxorubicin (17). The present study demonstrated the role of the lncRNA HOTAIR in mediating MDR of HCC cells by regulating $\mathrm{ABCB} 1$ expression, and revealed that the knockdown of HOTAIR increased chemosensitivity to cisplatin by suppressing STAT3/ABCB1 signaling.

lncRNAs have been identified as novel mediators of critical biological activities in hepatocarcinogenesis (7). IncRNA HOTAIR has been reported to be aberrantly upregulated in several types of cancer, including $\mathrm{HCC}$, and has roles in regulating the proliferation, apoptosis, invasiveness and metastasis of HCC $(12,13)$. The data of the present study validated that the knockdown of HOTAIR inhibited cell proliferation and migration of Huh7 cells, which was consistent with previous studies $(12,20)$. However, the association between HOTAIR and HCC MDR has rarely been reported. The present study identified that the knockdown of HOTAIR increased the chemosensitivity of Huh7 cells to cisplatin by reducing ABCB1 expression, indicating the role of HOTAIR in mediating MDR in HCC. These findings revealed that the knockdown of HOTAIR combined with cisplatin chemotherapy may serve as a potential therapeutic strategy to reverse MDR for HCC treatment. However, the mechanism by which HOTAIR regulates
$\mathrm{ABCB} 1$ expression is largely unknown. It has been confirmed that HOTAIR recruits PRC2 to regulate chromosome occupancy by enhancer of zeste 2 polycomb repressive complex 2 subunit (EZH2; a subset of PRC2), which leads to H3K27 trimethylation and the repression of multiple genes (7). It has been reported that inhibition of EZH2 may decrease the expression of $\mathrm{ABCB} 1$ as well as $\mathrm{ABC}$ subfamily $\mathrm{C}$ member 1 and ABC subfamily $G$ member 2 (junior blood group) mRNA, and protein expression levels $(21,22)$. Together with these findings, the present data indicated that HOTAIR may regulate ABCB1 expression in an EZH2-dependent manner. HOTAIR represses target gene expression, including Wnt inhibitory factor 1, by promoting histone $\mathrm{H} 3 \mathrm{~K} 27$ methylation in the promoter region via $\mathrm{EZH} 2$, and then activation of $\mathrm{Wnt} / \beta$-catenin signaling (23). However, data from the present and previous studies revealed that HOTAIR-knockdown or EZH2 inhibition may decrease ABCB1 expression $(21,22)$, indicating that HOTAIR regulated ABCB1 expression not via the EZH2-mediated H3K27 methylation of the $\mathrm{ABCB} 1$ promoter, but possibly through another mechanism.

Although the mechanism for transcriptional regulation of ABCB1 is not fully understood, a number of transcription factors, including Ras GTPase, SP1 transcription factor, nuclear factor- $\kappa$ B and STAT3 have been reported to be involved. STAT3, a transcription factor that participates in numerous cytokine 
signaling, has been demonstrated to be constitutively activated by tyrosine phosphorylation in HCC (24). Activation of STAT3 is associated with resistance of tumor cells to chemotherapeutic agents (25), and the downregulation of STAT3 is able to overcome chemoresistance (16). The present study identified that STAT3 activation had a mechanistic role in HOTAIR-mediated ABCB1 expression and MDR of HCC. Knockdown of HOTAIR reduced levels of p-STAT3 and increased chemosensitivity to cisplatin. Inhibition of STAT3 phosphorylation reduced HOTAIR-mediated ABCB1 expression in the present study. Furthermore, it has been reported that pSTAT3 directly mediates $\mathrm{ABCB} 1$ transcription, and regulates multidrug efflux in breast and ovarian cancer cells (19). Thus, the data from the present study may explain the association between HOTAIR and MDR of $\mathrm{HCC}$, and also revealed the possible underlying mechanism that HOTAIR mediated chemoresistance to cisplatin in HCC through activating the STAT3/ABCB1 signaling pathway. In addition, the findings of the present study added HOTAIR to the increasing list of upstream pathway leading to STAT3 activation, including interleukin-6, leukemia inhibitory factor, cytoplasmic tyrosine-protein kinase BMX and various receptor tyrosine kinases $(23,25)$. However, the molecular mechanism by which HOTAIR regulates STAT3 activation remains unclear. It has been reported that HOTAIR in laryngeal squamous cell cancer promoted the methylation of phosphatase and tensin homolog (PTEN), a negative regulator for phosphoinositide-3 kinase (PI3K) /RAC serine/threonine protein kinase (AKT) signaling pathway (26). HOTAIR-mediated PTEN methylation resulted in downregulation of PTEN mRNA and protein, leading to the activation of PI3K/AKT signaling. There are a number of negative regulators for STAT3 pathway, including suppressor of cytokine signaling 3 (SOCS3) (27); thus, further studies are required to confirm whether HOTAIR is able to regulate STAT3 activation through SOCS3 methylation.

In conclusion, the present study identified that HOTAIR-knockdown decreased cellular proliferation and cisplatin resistance of human Huh7 HCC cells. In addition, HOTAIR-knockdown resulted in a decrease in STAT3 activity and ABCB1 expression, and increased chemosensitivity to cisplatin. Furthermore, inhibition of STAT3 phosphorylation reduced HOTAIR-mediated ABCB1 expression and chemoresistance. The findings of the present study indicate that HOTAIR may have a role in mediating MDR by regulating STAT3/ABCB1 signaling, and identify HOTAIR as a potential novel therapeutic target to reverse MDR in HCC.

\section{Acknowledgements}

The present study was supported by The National Natural Science Foundation of China (grant no. 81000889), The Science and Technology Planning Project of Guangdong Province, China (grant no. 2014A020212094), The Natural Science Foundation of Guangdong Province, China (grant no. 2016A030313218), The Project of Sun Yat-Sen Memorial Hospital (grant no. YXQH201704), The Key Laboratory of Malignant Tumor Molecular Mechanism and Translational Medicine of Guangzhou Bureau of Science and Information Technology [grant no. (2013) 163] and The Key Laboratory of Malignant Tumor Gene Regulation and Target Therapy of Guangdong Higher Education Institutes (grant no. KLB09001).

\section{References}

1. Okuda K: Hepatocellular carcinoma. J Hepatol 32(Suppl 1): S225-S237, 2000.

2. Chiba T, Iwama A and Yokosuka O: Cancer stem cells in hepatocellular carcinoma: Therapeutic implications based on stem cell biology. Hepatol Res 46: 50-57, 2016.

3. Warmann S, Hunger M, Teichmann B, Flemming P, Gratz KF and Fuchs J: The role of the MDR1 gene in the development of multidrug resistance in human hepatoblastoma: Clinical course and in vivo model. Cancer 95: 1795-1801, 2002.

4. Zhuo L, Liu J, Wang B, Gao M and Huang A: Differential miRNA expression profiles in hepatocellular carcinoma cells and drug-resistant sublines. Oncol Rep 29: 555-562, 2013.

5. Roninson IB, Chin JE, Choi KG, Gros P, Housman DE, Fojo A, Shen DW, Gottesman MM and Pastan I: Isolation of human mdr DNA sequences amplified in multidrug-resistant KB carcinoma cells. Proc Natl Acad Sci USA 83: 4538-4542, 1986.

6. Ye CG, Yeung JH, Huang GL, Cui P, Wang J, Zou Y, Zhang XN, $\mathrm{He} \mathrm{ZW}$ and Cho CH: Increased glutathione and mitogen-activated protein kinase phosphorylation are involved in the induction of doxorubicin resistance in hepatocellular carcinoma cells. Hepatol Res 43: 289-299, 2013.

7. Mercer TR, Dinger ME and Mattick JS: Long non-coding RNAs: Insights into functions. Nat Rev Genet 10: 155-159, 2009.

8. Gupta RA, Shah N, Wang KC, Kim J, Horlings HM, Wong DJ, Tsai MC, Hung T, Argani P, Rinn JL, et al: Long non-coding RNA HOTAIR reprograms chromatin state to promote cancer metastasis. Nature 464: 1071-1076, 2010.

9. Rinn JL, Kertesz M, Wang JK, Squazzo SL, Xu X, Brugmann SA, Goodnough LH, Helms JA, Farnham PJ, Segal E and Chang HY: Functional demarcation of active and silent chromatin domains in human HOX loci by noncoding RNAs. Cell 129: 1311-1323, 2007.

10. Kim K, Jutooru I, Chadalapaka G, Frank J, Burghardt R, Kim S and Safe S: HOTAIR is a negative prognostic factor and exhibits pro-oncogenic activity in pancreatic cancer. Oncogene 32: 1616-1625, 2013.

11. Kogo R, Shimamura T, Mimori K, Kawahara K, Imoto S, Sudo T, Tanaka F, Shibata K, Suzuki A, Komune S, et al: Long noncoding RNA HOTAIR regulates polycomb-dependent chromatin modification and is associated with poor prognosis in colorectal cancers. Cancer Res 71: 6320-6326, 2011.

12. Fu WM, Zhu X, Wang WM, Lu YF, Hu BG, Wang H, Liang WC, Wang SS, Ko CH, Waye MM, et al: HOTAIR mediates hepatocarcinogenesis through suppressing miRNA-218 expression and activating P14 and P16 signaling. J Hepatol 63: 886-895, 2015.

13. Ishibashi M, Kogo R, Shibata K, Sawada G, Takahashi Y, Kurashige J, Akiyoshi S, Sasaki S, Iwaya T, Sudo T, et al: Clinical significance of the expression of long non-coding RNA HOTAIR in primary hepatocellular carcinoma. Oncol Rep 29: 946-950, 2013.

14. Gao JZ, Li J, DU JL and Li XL: Long non-coding RNA HOTAIR is a marker for hepatocellular carcinoma progression and tumor recurrence. Oncol Lett 11: 1791-1798, 2016.

15. Li H, An J, Wu M, Zheng Q, Gui X, Li T, Pu H and Lu D: LncRNA HOTAIR promotes human liver cancer stem cell malignant growth through downregulation of SETD2. Oncotarget 6: 27847-27864, 2015.

16. Real PJ, Sierra A, De Juan A, Segovia JC, Lopez-Vega JM and Fernandez-Luna JL: Resistance to chemotherapy via Stat3-dependent overexpression of Bcl-2 in metastatic breast cancer cells. Oncogene 21: 7611-7618, 2002.

17. Zhou JJ, Deng XG, He XY, Zhou Y, Yu M, Gao WC, Zeng B, Zhou QB, Li ZH and Chen RF: Knockdown of NANOG enhances chemosensitivity of liver cancer cells to doxorubicin by reducing MDR1 expression. Int J Oncol 44: 2034-2040, 2014.

18. Livak KJ and Schmittgen TD: Analysis of relative gene expression data using real-time quantitative PCR and the 2(-Delta Delta C(T)) method. Methods 25: 402-408, 2001.

19. Bourguignon LY, Peyrollier K, Xia W and Gilad E: Hyaluronan-CD44 interaction activates stem cell marker Nanog, Stat-3-mediated MDR1 gene expression and ankyrin-regulated multidrug efflux in breast and ovarian tumor cells. J Biol Chem 283: 17635-17651, 2008.

20. Wu Y, Zhang L, Zhang L, Wang Y, Li H, Ren X, Wei F, Yu W, Liu $\mathrm{T}$ and Wang $\mathrm{X}$ : Long non-coding RNA HOTAIR promotes tumor cell invasion and metastasis by recruiting EZH2 and repressing E-cadherin in oral squamous cell carcinoma. Int J Oncol 46: 2586-2594, 2015. 
21. Zhang Y, Liu G, Lin C, Liao G and Tang B: Silencing the EZH2 gene by RNA interference reverses the drug resistance of human hepatic multidrug-resistant cancer cells to 5-Fu. Life Sci 92: 896-902, 2013.

22. Fan TY, Wang H, Xiang P, Liu YW, Li HZ, Lei BX, Yu M and Qi ST: Inhibition of EZH2 reverses chemotherapeutic drug TMZ chemosensitivity in glioblastoma. Int J Clin Exp Pathol 7: 6662-6670, 2014.

23. Ge XS, Ma HJ, Zheng XH, Ruan HL, Liao XY, Xue WQ, Chen YB, Zhang Y and Jia WH: HOTAIR, a prognostic factor in esophageal squamous cell carcinoma, inhibits WIF-1 expression and activates Wnt pathway. Cancer Sci 104: 1675-1682, 2013.

24. Zhang L, Yang Z, Ma A, Qu Y, Xia S, Xu D, Ge C, Qiu B, Xia Q, Li J and Liu Y: Growth arrest and DNA damage 45G down-regulation contributes to Janus kinase/signal transducer and activator of transcription 3 activation and cellular senescence evasion in hepatocellular carcinoma. Hepatology 59: 178-189, 2014.
25. Bhardwaj A, Sethi G, Vadhan-Raj S, Bueso-Ramos C, Takada Y, Gaur U, Nair AS, Shishodia S and Aggarwal BB: Resveratrol inhibits proliferation, induces apoptosis and overcomes chemoresistance through down-regulation of STAT3 and nuclear factor-kappaB-regulated antiapoptotic and cell survival gene products in human multiple myeloma cells. Blood 109: 2293-2302, 2007.

26. Li D, Feng J, Wu T, Wang Y, Sun Y, Ren J and Liu M: Long intergenic noncoding RNA HOTAIR is overexpressed and regulates PTEN methylation in laryngeal squamous cell carcinoma. Am J Pathol 182: 64-70, 2013.

27. Ogata H, Chinen T, Yoshida T, Kinjyo I, Takaesu G, Shiraishi H, Iida M, Kobayashi T and Yoshimura A: Loss of SOCS3 in the liver promotes fibrosis by enhancing STAT3-mediated TGF-beta1 production. Oncogene 25: 2520-2530, 2006. 